[0212-7199(2003) 20: 1; pp 16-20]
ANALES DE MEDICINA INTERNA Copyright $\odot 2003$ ARAN EDICIONES, S.L.

AN MED INTERNA (Madrid) Vol. 20, N. ${ }^{\circ} 1$, pp. 16-20, 2003

\title{
Ocho casos de reumatismos asociados a cáncer
}

\author{
B. LLORENTE DÍEZ, J. L. ALONSO MARTÍNEZ, M. ECHEGARAY AGARA, \\ C. GONZÁLEZ ARENCIBIA, M. SOLANO REMÍREZ, \\ M. A. URBIETA ECHEZARRETA
}

Servicio de Medicina Interna. Hospital de Navarra. Pamplona. Navarra

EIGHT CASES OF RHEUMATISMS ASSOCIATED WITH CANCER

\begin{abstract}
RESUMEN
Objetivo: Estudiar las enfermedades reumáticas asociadas a cáncer diagnosticadas en un Servicio de Medicina Interna.

Material y métodos: Estudio descriptivo retrospectivo de los pacientes diagnosticados durante 1992 - 2000 de distintas enfermedades reumáticas asociadas a cáncer.

Resultados: De un total de 2127 pacientes se identificaron 8 casos de reumatismos paraneoplásicos en un periodo de tiempo de nueve años con una incidencia de $3,7 \%$, y un predominio de varones. El motivo de consulta en todos ellos fue la clínica de la enfermedad reumática. Seis de las ocho neoplasias fueron adenocarcinomas.

Conclusiones: Aunque los reumatismos paraneoplásicos no son frecuentes, es necesario tener en cuenta su existencia cuando existan antecedentes de neoplasias, en pacientes varones y cuando el curso clínico o la respuesta al tratamiento es atípica.
\end{abstract}

PALABRAS CLAVE: Neoplasia. Enfermedades reumáticas. Reumatismos paraneoplásicos.

\begin{abstract}
Objective: To study the rheumatic diseases associated with cancer diagnosed in an Internal Medicine Service.

Material and methods: A retrospective and descriptive study of the patients diagnosed during 1992 - 2000 of different rheumatic diseases associated with cancer.

Results: During a period of 9 years we identified 8 cases of paraneo plastic rheumatisms about a total of 2,127 patient, representing an inci dence of 3,7\%, with a predominance in males. The consultation motive in all them was the clinic of the rheumatic disease. Six of the eight neo plasias were adenocarcinomas.

Conclusions: Though the paraneoplastic rheumatisms are not fre quent, it is necessary take into account their existence when exist antece dent of neoplasia, in patient male and when the clinical course or res ponse to the treatment is atypical.
\end{abstract}

KEY WORDS: Neoplasias. Rheumatic disseases. Paraneoplastic rheu matisms.

Llorente Díez B, Alonso Martínez JL, Echegaray Agara M, González Arencibia C, Solano Remírez M, Urbieta Echezarreta MA. Ocho casos de reumatismo asociados a cáncer. An Med Interna (Madrid) 2003; 20: 16-20.

\section{INTRODUCCIÓN}

Las manifestaciones musculoesqueléticas del cáncer son aceptadas como entidades clínicas, aunque en general están basadas en series clínicas con un número pequeño de casos. Diversos tumores malignos pueden complicarse con la aparición de síndromes reumáticos y viceversa, enfermedades reumáticas durante su curso pueden complicarse con la aparición de una neoplasia $(1,2)$.

Las manifestaciones reumáticas asociadas a neoplasias pueden ser debidas a infiltración tumoral directa simulando una artritis (3-7) o a efectos remotos del cáncer, constituyendo éstos el verdadero reumatismo paraneplásico (SRP). La patogenia de los diferentes SRP se explica por uno o varios de los siguientes mecanismos: vía mediadores (hormonas, inmunoglobulinas $\mathrm{u}$ otros mediadores humorales) (8), vigilancia inmune alterada causando enfermedad reumática y neoplasia y efecto adverso de la terapéutica (9-11).

En general, se considera que un reumatismo es paraneoplásico cuando existe una relación temporal entre el trastorno reumático y el diagnóstico de la neoplasia, transcurriendo un tiempo no superior a 2 años entre ambos (2).

\section{MATERIAL Y MÉTODOS}

Presentamos 8 casos de diferentes enfermedades reumáticas asociadas a neoplasia diagnosticadas en nuestro Servicio de Medicina Interna entre los años 1992 -2000, extraídos de una revisión retrospectiva de un fichero informático donde se guardan datos clínicos, radiológicos, anatomopatológicos, y análisis, diagnóstico, tratamiento y seguimiento de los pacientes.

Trabajo aceptado: 16 de octubre de 2002

Correspondencia: Begoña Llorente Díez. Comunidad de Andalucía, 2-2º B. 31010 Barañain. Navarra 
Caso 1: Mujer de 59 años de edad diagnosticada en 1986 de car cinoma ductal infiltrante de mama derecha estadio $\mathrm{T}_{4}-\mathrm{N}_{\mathrm{x}}-\mathrm{M}_{0}$ que recibió tratamiento con quimioterapia, radioterapia pre-operatoria y mastectomia. Es evaluada en Septiembre- 1999 por artralgias con tumefacción de ambos tobillos de 2 meses de evolución. A la exploración presentaba artritis de ambos tobillos sin derrame articular. En la analítica de sangre destacaba Fibrinógeno: $572 \mathrm{mg} / \mathrm{dL}$; Fosfatasa alcalina elevada: 326 U/L; FR: $171 \mathrm{kU} / \mathrm{L}$, la serología de Salmonella y Brucella y los ANA fueron negativos. En RX de tobillos y pies: osteoblastosis parcheada de tobillo y pie izdos, con focos de hiperfijación en calota, esternón, parrillas, eje axial, sacro, sacroiliacas, hemipelvis izda, isquion, rodilla, tobillos y pies en gammagrafía ósea, sugestivos de metástasis. La paciente es diagnosticada de artritis por metástasis óseas de carcinoma de mama.

Caso 2: Varón de 58 años de edad que consultó en 1992 por artralgias de seis meses de evolución en muñecas, rodillas y articulaciones metacarpofalángicas con sinovitis en ambas muñecas y seis MCF. El FR era de $320 \mathrm{kU} / \mathrm{L}$ y se le apreciaron erosiones articulares en carpo izdo y $2^{\mathrm{a}} \mathrm{MCF}$ de mano dcha. La serología de Brucella, VHB, Lyme y los ANA fueron negativos, siendo diagnosticado de artritis reumatoide. Se inició tratamiento con Indometacina e inyecciones i.m. de sales de oro con mejoría de las artralgias, no progresión de las erosiones articulares y negativización del FR. Tras 24 meses de mejoría sin artritis vuelve a presentar artralgias en la misma localización y positivización del FR a título de $1200 \mathrm{kU} / \mathrm{L}$, con respuesta parcial al tratamiento con sales de oro e indometacina. Seguidamente comenzó a presentar pérdida de peso y dispepsia evidenciándose por Gastroscopia una lesión submucosa en antro con biopsia de adenocarcinoma de tipo indiferenciado difuso infiltrante. Se realizó cirugía del cáncer gástrico visualizándose metástasis hepáticas microscópicas en la biopsia hepática peroperatoria. El paciente falleció cuatro meses después del diagnóstico del adenocarcinoma de estómago.

Caso 3: Varón de 66 años de edad que ingresó en 1998 por dolor en rodillas y muslos de 4 meses de evolución que mejoraban con Diclofenaco. En el último mes inicia debilidad en ambas EEII, hiporexia, tos y expectoración mucoide, con pérdida de $3 \mathrm{kgs}$. A la exploración destacaba la presencia de hipoventilación en base pulmonar dcha, acropaquias en manos y pies y amiotrofia de cuadriceps precisando ayuda para incorporarse de una silla. Analíticamente presentaba anemia (Hb: 9,3 g/dL y Hcto: 28,9\%), VSG: $106 \mathrm{~mm} / \mathrm{h}(\uparrow)$; FNG: $790 \mathrm{mg} / \mathrm{dL}(\uparrow) ;$ PCR: $6,9 \mathrm{mg} / \mathrm{dL}(\uparrow)$. En el TAC torácico aparecía una masa de densidad sólida de $5 \mathrm{cms}$ de diámetro en LID con afectación de pleura visceral y derrame pleural dcho con biopsia obtenida por broncoscopia de adenocarcinoma poco diferenciado. En Gammagrafía ósea se apreció captación articular y periarticular predominante en rodillas sugestiva de osteoartropatía hipertrófica. El paciente fue diagnosticado de adenocarcinoma pulmonar y osteoartropatía hipertrófi $c a$.

Caso 4: Varón de 54 años de edad con antecedentes de colecistoduodenopancreatectomía cefálica por adenocarcinoma de ampolla de Vater en 1992. Ingresa en febrero-99 por dolores articulares en rodillas, tobillos, codos y muñecas de tipo inflamatorio de 40 días de evolución, y fiebre de $39^{\circ} \mathrm{C}$. En la anamnesis por aparatos destacaba la presencia de Raynaud en la mano derecha y pérdida de $4 \mathrm{Kgs}$ de peso. En la exploración física se evidenció la presencia de signos inflamatorios en rodillas, muñecas y tobillos con edema de ambas manos, sin signos de derrame ni hipertrofia sinovial. En la analítica de sangre se apreciaba fibrinógeno elevado: $747 \mathrm{mg} / \mathrm{dL}$; PCR: $21 \mathrm{mg} / \mathrm{dL}$ ( $\uparrow$ ); FR y ANA negativos. Serologias VHB, VHC, Brucella, Lyme, rubeola negativas. TAC abdominal masa retrogástrica con zonas de necrosis que desplazaba e infiltraba fundus y cuerpo gástrico con infiltración de pilar aórtico y posiblemente vena esplécnica. PAAF de masa retrogástrica por ecografía: positivo para adenocarcinoma. En RX de manos osteopenia yuxtaarticular en MCF sin erosiones. La clínica articular mejoró con Indometacina y Prednisona El paciente fue diagnosticado de poliartritis crónica simétrica seronegativa: síndrome RS3 PE y de adenocarcinoma de ampolla de Vater recidivado.

Caso 5: Varón de 62 años de edad, comenzó en mayo de 1993 con astenia, dolor en ambos hombros, dolor dorsal alto, en ingles y rodillas siendo detectada anemia. No presentó hinchazón articular, rigidez matutina, dolor basculante pelviano, fenómeno de Raynaud, aftas síndrome seco ni claudicación mandibular. En la exploración física presentaba dolor a la movilización de hombros, muñeca dcha, caderas, rodillas sin signos inflamatorios ni hipertrofia sinovial, siendo diagnosticado de polimialgia reumática que mejoró con tratamiento corticoideo. En septiembre de 1994 tras cuadro clínico de ictericia súbita indolora fue diagnosticado de colangiocarcinoma del que fue intervenido, estando asintomático en la actualidad. El paciente fue diagnosticado de Polimialgia Reumática probablemente como presentación paraneoplásica de colangiocarcinoma.

Caso 6: Mujer de 49 años de edad, ingresa en enero de 1994 refiriendo desde hacia dos años lesiones cutáneas de inicio en palmas y dedos de las manos con posterior extensión a la cara, con edema y enrojecimiento palpebral. Desde hacia un mes refería síndrome seco. Desde el inicio del cuadro notaba debilidad muscular y sensación de hinchazón en brazos desde los hombros hasta las manos, había sido tratada con 6-m-prednisolona con mejoría parcial. En la exploración física presentaba lesiones cutáneas de varios tipos afectando a piel expuesta y cubierta: eritema en pared abdominal, dorso y muslos, placas induradas en pulpejos de los dedos, eritema periungueal, pápulas de Gottron y edema palpebral bilateral con eritema no violáceo. Fuerza muscular $3+/ 5$ en cintura escapular, pelviana y extensores del cuello. Gowers positivo. En analítica de sangre destacaba VSG: $43 \mathrm{~mm} / \mathrm{h}(\uparrow)$; GOT: 102 U/L ( $\uparrow$ ); GPT: 54 U/L ( $\uparrow) ;$ LDH: 784 U/L ( $\uparrow)$; CPK: 1585 U/L ( $\uparrow$ ). Biopsia de piel y músculo deltoides: atrofia muscular focal, con infiltrado intercelular por macrófagos que están digiriendo el músculo con capilaritis y afectación vascular de mayor tamaño por células musculares. Fascia normal. Electromiograma compatible con afectación mixta neurógena y muscular. Se diagnostica de Dermatomiositis y se inicia tratamiento con esteroides y Azatioprina con mejoría parcial de la fuerza hasta $4 / 5$ permitiendo vida autónoma de la paciente. Seis meses después del diagnóstico se objetiva nódulo mamario que se biopsia con resultado de adenocarcinoma de mama. Se realiza tumorectomía, y tratamiento oncológico quimioradioterápico falleciendo 18 meses después por enfermedad metastásica incontrolable. Fue diagnosticada de Dermatomiositis y adenocarcinoma de mama.

Caso 7: Varón de 59 años de edad, visto en mayo-1994 por episodios de febrícula intermitentes desde 1991, que se alternaban con edemas en los tobillos de forma secuencial, sin enrojecimiento ni impotencia funcional. En abril-1996 continua con febrícula prolongada, junto con dolor en extremidades y esqueleto axial en los últimos meses, hipoacusia, acúfenos y fenómeno de Raynaud en primer dedo de mano izda.

A finales de abril de 1996 el paciente presenta de forma súbita dolor intenso y tumefacción en cartílago auricular (trago) de pabellón auricular izdo y dolor y tumefacción de párpado superior de ojo izdo con fiebre de $38-38,5^{\circ} \mathrm{C}$. Había presentado en los dos años anteriores otros dos episodios de distribución bilateral y episodios agudos de disfonia. No presentaba dolor ni signos inflamatorios laríngeos ni de nariz. En la exploración física aparecía eritema, tumefacción y dolor en trago izdo. Cartílagos de nariz y laríngeos no dolorosos. Disminución de la hendidura palpebral izda por tumefacción de párpado superior. Induración cutánea y subcutánea en región posterior de pierna izda con equimosis punteada superficial. El paciente es diagnosticado en este momento de Policondritis recidivante, iniciándose tratamiento con AINEs y con esteroides.

En febrero de 1997 refiere dolor dorsal nocturno irradiado hacia esternón. A la exploración presenta maniobra de Schöber de 7 a 11,5 cms y rigidez dorsal a la lateralización. En RX de sacroiliacas se 
aprecia sacroileitis grado II, siendo diagnosticado además de espodi loartropatia seronegativa HLA B27 positivo.

En octubre-1998 presenta astenia, dolor epigástrico, heces melénicas y un nuevo episodio de dolor e inflamación en pabellón auricular dcho. En la analítica de sangre: Hb. 9,4 g/dL; Hcto: 28,3\%; VCM: 89,2 $\mu \wedge 3$; VSG: 85 mm/h; FNG: 766 mg/dL; PCR: $18 \mathrm{mg} / \mathrm{dL}$; CEA: 284,7 $\mu \mathrm{g} / \mathrm{L} ; \mathrm{Ca}$ 19,9: $698 \mathrm{kU} / \mathrm{L}$. En la colonoscopia presencia de neoplasia de colon ascendente, con biopsia de adenocarcinoma con metástasis hepáticas y pulmonares en TAC. El paciente finalmente fue diagnosticado de Adenocarcinoma de colon con metástasis hepáticas y pulmonares, policondritis recidivante y espondiloartritis seronegativa. Se realizó hemicolectomía derecha y tratamiento con 5-fluoracilo y ácido folínico con progresión de las metástasis, siguiendo posteriormente tratamiento con Irinotecan, falleciendo en abril-2000.

Caso 8: Paciente varón de 76 años con antecedentes de gastrectomia parcial hacia 1980 por ulcus duodenal, ingresado en julio-2000 cefalea, claudicación mandibular de 3 meses de evolución y antes del ingreso disminución brusca de visión de ojo derecho. A la exploración física destacaba engrosamiento de arterias temporales y de arteria facial derecha. En analítica de sangre destaca la presencia de VSG elevada $(68 \mathrm{~mm} / \mathrm{h})$. En la biopsia de arteria temporal realizada aparece arteritis de células gigantes iniciándose tratamiento con prednisona con mejoría sintomática. En el mismo mes el paciente presenta HDA, y en gastroscopia realizada aparece ulcera gástrica de aspecto benigno. En septiembre de 2000 y tras realización de gastroscopia de revisión y biopsias de rutina se encuentra adenocarcinoma gástrico por lo que se realiza gastrectomia total en noviembre de 2000. El paciente finalmente fue diagnosticado de Adenocarcinoma gástrico y Arteritis de células gigantes.

\section{RESULTADOS}

De un total de 2127 pacientes correspondientes a un área clínica de 8 camas de un hospital terciario, se identificaron 8 casos de reumatismo paraneoplásico en un periodo de 9 años con una incidencia de $3,7 \%$.
Las características clínicas de los 8 pacientes en el estudio están resumidas en la Tabla I. Seis eran varones $(75 \%)$ y 2 mujeres (25\%), con edad media de $60 \pm 8$ años, rango de 49 a 76 años.

En todos los casos el motivo de consulta fue la clínica de enfermedad reumática, estando algunos diagnosticados previamente de la neoplasia (caso 1, caso 4). En el resto el diagnostico se realizó a lo largo de la evolución de la enfermedad reumática.

El intervalo entre la presentación de la enfermedad reumática y el diagnóstico de la neoplasia fue variable, en dos de los siete casos (caso 1 y caso 4) la neoplasia se diagnosticó previamente, en el caso 1 el intervalo fue de 13 años y en el caso 4 fue de 7 años, aunque en ambos coincidió con la recidiva del tumor. En el caso 3 ambas entidades se manifestaron al mismo tiempo. En el caso 8 la neoplasia apareció 2 meses después del diagnóstico de la enfermedad reumática. En el resto de casos la enfermedad reumática se manifestó antes que la neoplasia con un intervalo de 1 a 2 años entre ambos.

El caso 1 puede ser considerado como invasión del hueso por el tumor, mientras que el resto corresponden a verdaderos SRP. Ninguno de los casos corresponden al grupo de disrregulación inmune en el curso de una enfermedad reumatológica ni reacciones adversas de la terapia antitumoral.

Sólo dos $(25 \%)$ de los ocho casos (caso 1 y caso 2 ) presentaron pruebas serológicas reumáticas positivas y en ambas fue el factor reumatoide.

Seis de las ocho neoplasias $(75 \%)$ fueron adenocarcinomas en la anatomía patológica: cuatro del aparato digestivo (dos gástricos, uno de colon y uno de ampolla de Vater), uno de pulmón y uno de mama, un carcinoma de mama (25\%) y un Colangiocarcinoma (25\%).

Los pacientes fueron tratados en su mayoría inicialmente para la enfermedad reumatológica con antiinflamatorios no esteroideos (Diclofenaco e Indometacina), corticoides (Pred-

TABLA I

ENFERM EDADES REU M ÁTICAS ASO CIADAS A NEO PLASIAS

\begin{tabular}{|c|c|c|c|c|c|}
\hline Paciente & Edad/Sexo & Enfermedad reumática & Neoplasia asociada & Serología & $\begin{array}{l}\text { Tratamiento para la } \\
\text { enfermedad reumática }\end{array}$ \\
\hline 1 & $59 / M$ & Artritis por metástasis óseas & $\begin{array}{l}\text { Carcinoma ductal } \\
\text { infiltrante de mama }\end{array}$ & $\begin{array}{l}\text { FR: } 171(\uparrow) \\
\text { ANA: }(-)\end{array}$ & Prednisona \\
\hline 2 & $58 / \mathrm{V}$ & Artritis reumatoide & $\begin{array}{l}\text { ADC gástrico con } \\
\text { metástasis hepáticas }\end{array}$ & $\begin{array}{c}\text { FR: } 320(\uparrow) \rightarrow 1200 \\
\text { ANA: }(-)\end{array}$ & $\begin{array}{l}\text { Indometacina } \\
\text { y sales de oro }\end{array}$ \\
\hline 3 & $66 / \mathrm{V}$ & O steoartropatia hipertrófica & ADC de pulmón & - & Diclofenaco \\
\hline 4 & $54 / \mathrm{V}$ & $\begin{array}{l}\text { Poliartritis crónica simétrica } \\
\text { (síndrome RS3 PE) }\end{array}$ & $\begin{array}{l}\text { ADC de ampolla } \\
\text { de Vater }\end{array}$ & $\begin{array}{c}\text { FR: }(-) \\
\text { ANA:(-) } \\
\text { ANCAs:(-) }\end{array}$ & $\begin{array}{l}\text { Indometacina } \\
\text { Prednisona }\end{array}$ \\
\hline 5 & $62 / \mathrm{V}$ & Polimialgia reumática & Colangiocarcinoma & ANAs:(-) & Prednisona \\
\hline 6 & $49 / M$ & Dermatomiositis & ADC de mama & - & $\begin{array}{l}\text { Azatioprina } \\
\text { Prednisona }\end{array}$ \\
\hline 7 & $59 / \mathrm{V}$ & $\begin{array}{c}\text { Policondritis recidivante y } \\
\text { espondiloartritis seronegativa }\end{array}$ & $\begin{array}{l}\text { ADC de colon con } \\
\text { metástasis hepáticas } \\
\text { y pulmonares }\end{array}$ & $\begin{array}{c}\text { FR:(-) } \\
\text { ANAs:(-) } \\
\text { ANCAs:(-) }\end{array}$ & $\begin{array}{l}\text { Indometacina } \\
\text { Prednisona }\end{array}$ \\
\hline 8 & $76 / V$ & Arteritis de células gigantes & ADC gástrico & - & Prednisona \\
\hline
\end{tabular}


nisona) y uno de ellos con Sales de Oro (caso 2), con mejoría de la sintomatología reumatológica en mayor o menor grado.

\section{DISCUSIÓN}

El curso clínico de los tumores malignos puede verse complicado por la aparición de diversos SRP y viceversa, enfermedades reumáticas en su curso pueden ser complicados por la aparición de neoplasias $(1,2)$.

Las manifestaciones musculoesqueléticas asociadas al cáncer obedecen a diferentes mecanismos patogénicos: a) invasión de hueso, articulación o músculo por el tumor (3-7) (caso 1 de nuestro estudio); b) síndromes paraneoplásicos inducidos a distancia del tumor vía mediadores (8) (casos 2-8 de nuestro estudio); c) vigilancia inmune alterada causando ambas enfermedades reumáticas y enfermedades neoplásicas (9-11) (ningún caso en nuestro estudio); y d) reacciones adversas de terapia anticancerosa $(12,13)$ (ningún caso en nuestro estudio).

Tumores primarios de hueso y tejidos blandos, así como metástasis que afectan al tejido articular y yuxtaarticular, pueden causar una reacción inflamatoria sinovial mediada por citoquinas proinflamatorias segregadas por células tumorales y por la actividad de macrófagos, linfocitos y neutrófilos que envuelven la reacción desmoplástica yuxtatumoral $(6,7)$ pudiendo manifestarse como una monoartritis, afectando sobre todo a la rodilla.

SRP son aquellos síndromes reumáticos asociados al cáncer que ocurren a distancia del tumor primario y son inducidas por el cáncer a través de hormonas, péptidos, mediadores autocrinos o paracrinos, anticuerpos y linfocitos citotóxicos. Pueden preceder (no más de 2 años) (casos 2, 5, 6, 7 y 8) al diagnóstico de la neoplasia; ser marcadores de recidiva tumoral (casos 1 y 4 de nuestro estudio) o aparecer concomitantemente (caso 3 de nuestro estudio) o después del diagnóstico del tumor $(1,2,6,7,13,14)$ (ningún caso en nuestro estudio). El curso clínico de los SRP es habitualmente paralelo al del tumor. De este modo, la curación de la neoplasia usualmente, aunque no invariablemente, resulta en la regresión del SRP. Han sido descritas remisiones espontáneas de SRP pero también la persistencia a pesar de la "curación" del cáncer $(15,16)$.

El reconocimiento de la enfermedad paraneoplásica es clínicamente importante, ya que su aparición puede ser el primer signo de una neoplasia maligna. Además los SRP pueden complicar el estadiaje del cáncer simulando enfermedad metastásica; por otra parte las verdaderas metástasis pueden comportarse como SRP y ser ofertada erróneamente una cirugía radical (18-21).

La valoración de la naturaleza paraneoplásica del síndrome reumático constituye un desafío médico, ya que las manifestaciones reumáticas del cáncer son usualmente indistinguibles de las enfermedades reumáticas idiopáticas y el diagnóstico de la naturaleza paraneoplásica sólo es posible tras el diagnóstico del cáncer (casos 2, 5, 6, 7, 8). La lista de síndromes paraneoplásicos reumáticos se ha visto incrementada en revisiones recientes (15). El diagnóstico del cáncer a través de síndromes paraneoplásicos reumáticos (Tabla II) precisa de un alto índice de sospecha basado en el conocimiento de las asociaciones clínicas. Aunque el comportamiento clínico de dichos síndromes paraneoplásicos puede ser
TABLA II

CLASIFICACIÓ N DE CÁNCER ASO CIADO A SÍNDROM ES REUM ÁTICOS

\begin{tabular}{l}
\hline Artropatías \\
\hline Afectación articular local por Cáncer. \\
Poliartritis - Cáncer. \\
O steoartropatía hipertrófica. \\
Polimialgia reumática y síndrome polimiálgico atípico. \\
Fascitis palmar y artritis. \\
Gota. \\
Poliartritis recidivante. \\
Polisinovitis edematosa benigna. \\
Sacroileitis. \\
Enfermedad de Still del adulto. \\
\hline Enfermedades musculares \\
\hline Dermatomiositis, polimiositis y dermatomiositis sin \\
miositis. \\
M iositis nodular localizada. \\
M iopatía necrotizante. \\
Síndrome miasténico Lamberg-Eaton. \\
\hline Esclerodermia, Paniculitis y Fascitis
\end{tabular}

Esclerodermia, Paniculitis y Fascitis

Esclerosis sistémica.

Fascitis eosinófila.

Eritema nodoso.

Paniculitis-artritis.

Vasculitis

Miscelánea

Distrofia simpática refleja.

Síndrome de Sjögren.

O steomalacia.

Hiperostosis esquelética.

Síndrome antifosfolípido.

Crioglobulinemia.

totalmente típico, deberemos sospechar la existencia de cáncer asociado cuando tengan unas manifestaciones clínicas atípicas y la respuesta al tratamiento no sea la esperada.

En nuestro estudio la mayoría de tumores relacionados con SRP son adenocarcinomas (6 de los 8 casos encontrados), siendo la localización más frecuente en el aparato digestivo. Son más frecuentes en varones ( 6 de los 8 casos del estudio) y en las 2 mujeres del estudio se relaciona con presencia de neoplasia mamaria.

El cáncer puede aparecer en el curso de ciertas enfermedades reumáticas como resultado de la disrregulación inmune. La transformación de la autoinmunidad en malignidad ha sido bien estudiada en el síndrome de Sjögren y la linfadenopatía angioinmunoblástica, pero también puede operar en el Lupus eritematoso sistémico, Artritis reumatoide y deficiencia genéticamente determinada de Ig A y su asociación con tumores linfoproliferativos $(2,15-17)$. El defecto básico de estas enfermedades puede estar en la deficiencia de la función de las células $\mathrm{T}$ supresoras (11). Esta alternativa puede conducir a la proliferación incontrolada de células linfoides $\mathrm{B}$ y después a la transición de hiperplasia linfoide policlonal a la enfermedad prolife- 
rativa monoclonal requiriendo varios años para desarrollarse. Por lo tanto, el intervalo entre el diagnóstico de la enfermedad reumática y el diagnóstico de malignidad puede ser tan largo como 20 años, en contraste con el relativo corto intervalo entre el SRP y el diagnóstico de su neoplasia asociada (2).

Ciertas drogas usadas en el tratamiento de enfermedades reumáticas conllevan un alto riesgo de desarrollar enfermedades malignas específicas. En pacientes con Artritis reumatoide tratados con Ciclofosfamida existe $1,5 \%$ de riesgo relativo de cáncer y el desarrollo de linfoma en pacientes tratados con altas dosis de Metotrexate y Ciclosporina A han sido descritos. Se ha sugerido que las drogas citotóxicas pueden promover un estado de permisividad inmunológico para el desarrollo de linfomas en población susceptible.

En resumen, aunque infrecuentes, las manifestaciones reumáticas del cáncer manifestadas como diferentes síndromes paraneoplásicos deben ser tenidas en cuenta y pueden ayudar en el diagnóstico temprano de estos pacientes: El inicio de una poliartritis asimétrica en el anciano de comienzo agudo, la presencia de gammapatía monoclonal en pacientes con artritis reumatoide o síndrome de Sjögren, el desarrollo de osteoartropatía hipertrófica, la dermatomiositis, el síndrome fascitis palmar y artritis, paniculitis de curso crónico, polimialgia remática con signos atípicos y la existencia de vasculitis leucocitoclástica en edades superiores a 50 años sin otra causa explicativa deben hacer sospechar la coexistencia o el desarrollo posterior de una neoplasia.

En general, debemos sospechar la existencia de reumatismo paraneoplásico en aquellos pacientes con antecedentes clínicos de cáncer, planteándonos la recidiva tumoral como causa, y también en aquellos pacientes que se presenten con cuadros reumáticos de comportamiento clínico atípico o que muestren resistencia al tratamiento, particularmente en varones.

\section{Bibliografía}

1. Caldwell DS, McCallum RM: Rheumatologic manifestations of cancer. Med Clin North Am 1986; 70: 385-417.

2. Naschitz JE, Rosner I, Rozenbaum M, Zuckerman E, Yeeshurun D. Rheumatic Syndromes: Clues to occult neoplasia. Semin Arthritis Rheum 1999; 29: 43-55.

3. Lagier R. Synovial reaction caused by adjacent malignant tumors: anatomicopathological study of three cases. J Rheumatol 1977; 4: 65-72.

4. Shenberger KN, Morgan GJ jr. Recurrent malignant melanoma presenting as monoarthritis. J. Rheumatol 1982; 9: 328.

5. Schwarzer AC, Fryer J, Preston SJ, Schrieber L, McNaught PJ. Metastatic adenosquamous carcinoma presenting as an acute monoarthritis, with review of literature. J Orthop. Rheum 1990; 3: 175-85.

6. Brooks PM: Rheumatic manifestations of neoplasia. Curr Opin Rheumatol 1992; 4: 90-93.

7. Butler RC, Thompson JM. Keat ACS: Paraneoplasic rheumatic disorders: a review. J Royal Soc Med1987; 80: 168-72.

8. Minna JD, Bunn PA Jr. Paraneoplastic syndromes, in DeVita VT Jr. Helman S, Rosemberg SA (eds). Cancer: principles and practice of oncology. Philadeophia, PA, Lippincott 1982. p. 1476-517.

9. Sela O, Shoenfeld Y: Cancer in autoinmune diseases. Semin Arthritis Rheum 1988;18: 77-87.

10. Roubinian JR, Talan N. Neoplasia, autoimmunity and the immune response, Adv Int Med 1978; 23: 435-50.

11. Abdou NI, Lindley HB, Racela LS. Supressor T-cell disfunction and anti-supressor cell antibody in active early rheumatoid arthritis. J. Rheumatol 1981; 8: 9-18.

12. Naschitz JE, Abrahamsom J, Yeshurun D. The clinical significance of paraneoplasic syndrome. Oncology 1989; 46: 40-5.

13. Buchanan WW. Systemic disorders with rheumatic manifestations. Curr Opin Rheumatol. 1994; 6: 51-4.

14. Conaghan PG, et al. Rheumatic manifestations of malignancy. Curr Opin Rheumatol. 1994; 6: 105-10.

15. Naschitz JE, Rosner I, Rozenbaum M, Elias N, Yeeshurun D. Cancerassociated rheumatic disorders: clues to occult neoplasia. Semin Arthritis Rheum 1995; 24: 231-41.

16. Naschitz JE, et al. Rheumatic manifestations of occult cancer. Cancer 1995; 75: 2954-58.

17. Seda H, et al. Musculoskeletal syndromes associated with malignances. Curr Opin Rheumatol 1995;24:76-78.

18. Avina-Zubieta JA, Enkerlin HL, Galindo-Rodriguez G. Rheumatic manifestations of malignancy. Curr Opin Rheum 1996; 47-51.

19. Carsons $\mathrm{S}$. The association of malignancy with rheumatic and connective tissue disseases. Semin Oncol 1997; 24: 360-72.

20. Hall TC. Paraneoplasic syndromes: mechanisms. Semin Oncol 1997; 24: 269-75.

21. Nathanson L, Hall TC. Introduction: paraneoplasic syndromes. Semin Oncol 1997; 24: 265-8. 